\title{
Gallium Nitrate
}

National Cancer Institute

\section{Source}

National Cancer Institute. Gallium Nitrate. NCI Thesaurus. Code C1109.

A hydrated nitrate salt of the group IIla element gallium with potential use in the treatment of malignancy-associated hypercalcemia. Gallium nitrate localizes preferentially to areas of bone resorption and remodeling and inhibits osteoclastmediated resorption by enhancing hydroxyapatite crystallization and reduction of bone mineral solubility. This agent also increases calcium and phosphorous deposition into bone and may increase collagen synthesis. ( $\mathrm{NCl04)}$ 